\title{
STABLE DISCRETIZATION METHODS WITH EXTERNAL APPROXIMATION SCHEMES
}

\author{
RAM U. VERMA \\ International Publications \\ 12046 Coed Drive \\ Orlando, Florida 32826, USA
}

(Received September, 1994; Revised May, 1995)

\begin{abstract}
We investigate the external approximation-solvability of nonlinear equations - an upgrade of the external approximation scheme of Schumann and Zeidler [3] in the context of the difference method for quasilinear elliptic differential equations.
\end{abstract}

Key words: External Approximation Scheme, Approximation-Solvability, Difference Method.

AMS (MOS) subject classifications: $65 \mathrm{~J} 15,47 \mathrm{H} 17$.

\section{Introduction}

Based on the inner approximation schemes of Petryshyn [1, 2] for projection methods, Schumann and Zeidler [3] applied an external approximation scheme to difference method for quasilinear elliptic differential equations. Here we generalize the approximation-solvability of nonlinear operator equations corresponding to an external approximation scheme, which upgrades the external approximation of Schumann and Zeidler. Finally, we consider an application to the abstract generalization.

For details on the approximation-solvability, see [1-5].

Next, let $\pi_{0}=\left\{X, F, X_{n}, X^{*}, X_{n}^{*}, A, W, A_{n}, R_{n}, K_{n}, E_{n}\right\}$ be an external approximation scheme represented by a diagram

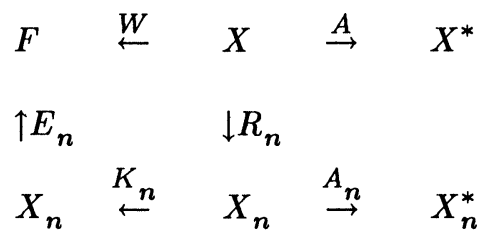

where $X, F, X_{n}$ are real Banach spaces with $F$ reflexive and $\operatorname{dim} X_{n}<\infty$ for all $n \in N$. Here $R_{n}: X \rightarrow X_{n}$ is a restriction operator, $E_{n}: X_{n} \rightarrow F$ is an extension operator, $K_{n}: X_{n} \rightarrow X_{n}$ is a linear continuous operator, and $W: X \rightarrow F$ is a synchronization operator. All operators $R_{n}, K_{n}$ and $E_{n}$ are linear and continuous with $\sup \left\|R_{n}\right\|<\infty, \sup \left\|K_{n}\right\|<\infty$ and $\sup \left\|E_{n}\right\|<\infty$. The operator $W$ is linear, continuous and injective. Furthermore, all operators $A_{n}$ are continuous.

The approximation scheme $\pi_{0}$ coincides with the following external approximation scheme of Schumann and Zeidler [3], $\pi_{1}=\left\{X, F, X_{n}, X^{*}, X_{n}^{*}, A, W, A_{n}, R_{n}, E_{n}\right\}$ when $K_{n}$ is the 
identity:

$$
\begin{array}{lllll}
F & \stackrel{W}{\leftarrow} & X & \stackrel{A}{\rightarrow} & X^{*} \\
E_{n} \uparrow & & \downarrow R_{n} & & \\
& & X_{n} & \stackrel{A_{n}}{\rightarrow} & X_{n}^{*}
\end{array}
$$

and $\pi_{0}$ reduces to the inner approximation schemes of Petryshyn [1,2] for projection methods when $F=X$, and $W$ and $K_{n}$ are the identities.

Let us recall some definitions for the sake of the completeness. In what follows, the symbols “ $\rightarrow$ " and $~ \stackrel{w}{\rightarrow}$ " shall denote the strong convergence and weak convergence, respectively.

D1.1 (Admissible external approximation scheme): The approximation scheme $\pi_{0}$ is called an admissible external approximation scheme if the following implications should hold:

(I1) Compatibility condition: For all $x \in X$, as $n \rightarrow \infty$,

$$
E_{n} K_{n} R_{n} x \rightarrow W(x) \text { in } F \text {. }
$$

(I2) Synchronization condition: The weak limits in $F$ of the sequences $\left\{E_{n} K_{n} x_{n}\right\}$ and their subsequences are synchronized, that is, if

$$
E_{n^{\prime}} K_{n^{\prime}} x_{n^{\prime}} \stackrel{w}{\rightarrow} f \text { in } F \text { as } n \rightarrow \infty
$$

then $f \in W(X)$.

D1.2 (Discrete convergence): For a sequence $\left(x_{n}\right)$ of elements with $x_{n} \in X_{n}$ for all $n \in N,\left(x_{n}\right)$ is said to converge discretely to $x\left(x_{n} \stackrel{d}{\rightarrow} x\right)$ iff

$$
\lim _{n \rightarrow \infty}\left\|x_{n}-R_{n} x\right\|=0
$$

D1.3 (Discrete ${ }^{*}$ convergence): For a sequence $\left(x_{n}^{*}\right)$ of functionals with $x_{n}^{*} \in X_{n}^{*}$ for all $n \in N$, the sequence $\left(x_{n}^{*}\right)$ is said to converge discretely* to $x^{*} \in X^{*}\left(x_{n}^{*} \stackrel{d^{*}}{\rightarrow} x^{*}\right)$ iff

$$
\lim _{n \rightarrow \infty}\left[x_{n}^{*}, x_{n}\right]_{X_{n}}=\left[x^{*}, x\right]_{X}
$$

holds for all sequences $\left(x_{n}\right), x_{n} \in X_{n}$ with sup $\left\|x_{n}\right\|_{X_{n}}<\infty$ and

$$
E_{n} K_{n} x_{n} \stackrel{w}{\rightarrow} W(x) \text { in } F \text { as } n \rightarrow \infty \text {. }
$$

\section{External Approximation-Solvability}

In this section, we consider the unique approximation-solvability of the initial value problem

$$
A x=b, \quad x \in X,
$$

and corresponding discretized problem 


$$
A_{n} x_{n}=b_{n}, \quad x_{n} \in X_{n}, \quad n=1,2, \ldots
$$

with respect to the approximation scheme $\pi_{0}$ represented by the diagram (1).

Theorem 2.1: $\quad$ Suppose that the approximation scheme $\pi_{0}$ represents an admissible external approximation scheme, and the following assumptions hold:

(A1) Weak Consistency: For all $x \in X$,

$$
A_{n} R_{n} x \stackrel{d^{*}}{\rightarrow} A x .
$$

(A2) Stability: For all $x, y \in X_{n}$ and $n \geq n_{0}$,

$$
\left\|A_{n} x-A_{n} y\right\|_{X_{n}^{*}} \geq \mu\left(\|x-y\|_{X_{n}}\right)
$$

where $\mu$ is a suitable gauge function.

(A3) Approximation of the term $b$ in (3): For each $b \in X^{*}$, there exists a sequence $\left(b_{n}\right)$ such that

$$
b_{n} \stackrel{d^{*}}{\rightarrow} b \text { for } b_{n} \in X_{n}^{*} \text { and for all } n \geq n_{0} .
$$

Then the following conditions are equivalent:

(C1) Solvability: For each $b \in X^{*}$, the equation

$$
A x=b, x \in X
$$

has a solution.

(C2) Unique approximation-solvability: The equation $A x=b$ is said to be uniquely approximation-solvable if the following implications hold:

(i) For $b \in x^{*}$, the equation $A x=b$ has a unique solution $x \in X$.

(ii) For each $b_{n} \in X_{n}^{*}$ and all $n \geq n_{0}$, the approximate equation

$$
A_{n} x_{n}=b_{n}
$$

(iii) As $n \rightarrow \infty$,

has a unique solution $x_{n} \in X_{n}$.

$$
b_{n} \stackrel{d^{*}}{\rightarrow} b \Rightarrow x_{n} \stackrel{d}{\rightarrow} x \text { and } E_{n} K_{n} x_{n} \rightarrow W(x) \text { in } F .
$$

(C3) A-properness: The operator $A: X \rightarrow X^{*}$ is A-proper with respect to the approximation scheme $\pi_{0}$, that is, if the following implications hold:

$$
A_{n^{\prime}} x_{n^{\prime}} \stackrel{d^{*}}{\rightarrow} b \text { and } \sup \left\|x_{n^{\prime}}\right\|_{X_{n^{\prime}}}<\infty
$$

imply the existence of a subsequence $\left(x_{n^{\prime \prime}}\right)$ such that

$$
x_{n^{\prime \prime}} \stackrel{d}{\rightarrow} x \text { and } A x=b .
$$

More precisely, the theorem can be expressed as follows: If the approximation scheme $\pi_{0}$ is an admissible external approximation scheme with weak consistency and stability, then the equation $A x=b, x \in X$, is uniquely approximation-solvable iff $\boldsymbol{A}$ is $\boldsymbol{A}$-proper.

Remark 2.2: Let the assumptions $(A 1)-(A 3)$ hold. Then we have two different situations for using Theorem 2.1: 
(S1) Abstract existence theorems imply the unique approximation-solvability, that is, if the equation $A x=b, x \in X$, has a solution, i.e., $(C 1)$ holds, then, by Theorem 2.1 , the equation $A x=b$ is uniquely approximation-solvable, and $A: X \rightarrow X^{*}$ is $A$-proper.

(S2) $A$-properness implies the unique approximation-solvability, that is, if we show the $A$ properness of $A: X \rightarrow X^{*}$ by a direct argument, then the equation $A x=b, x \in X$, by Theorem 2.1 , is uniquely approximation-solvable.

Corollary 2.3: Theorem 2.1 reduces to the theorem of Schumann and Zeidler [3] when $K_{n}$ is the identity.

Before proving Theorem 2.1, we give a lemma, crucial to the proof.

Lemma 2.4: Let $\pi_{0}$ be an admissible external approximation scheme. Then the following implications hold:

(ii) $\quad x_{n}^{*} \stackrel{d^{*}}{\rightarrow} 0 \Rightarrow \lim _{n \rightarrow \infty}\left\|x_{n}^{*}\right\|=0$.

Proof: $(i)$ Let $x_{n}^{*} \stackrel{d^{*}}{\rightarrow} x^{*}$. Assume $\sup _{n}\left\|x_{n}^{*}\right\|<\infty$ does not hold. Then there is a subsequence, again denoted by $\left(x_{n}^{*}\right)$, such that

$$
\left\|x_{n}^{*}\right\|>n \text { for all } n .
$$

As $\left\|x_{n}^{*}\right\|=\sup \left\{\left[x_{n}^{*}, x_{n}\right]:\left\|x_{n}\right\|=1\right\}$, there exists a subsequence, again denoted by $\left(x_{n}\right)$, such that

$$
\left\|x_{n}\right\|=1 \text { and }\left[x_{n}^{*}, x_{n}\right]>n \text { for all } n \text {. }
$$

Since $\sup _{n}\left\|E_{n}\right\|<\infty$ and $\sup _{n}\left\|K_{n}\right\|<\infty$, we have $\sup _{n}\left\|E_{n} K_{n} x_{n}\right\|<\infty$. Given that $F$ is reflexive,

$$
E_{n} K_{n} x_{n} \stackrel{w}{\rightarrow} f \text { in } F \text { as } n \rightarrow \infty .
$$

The synchronization condition (I2) implies that

$$
f=W(x) .
$$

Thus, $x_{n}^{*} \stackrel{d^{*}}{\rightarrow} x^{*}$ leads to

$$
\left[x_{n}^{*}, x_{n}\right] \rightarrow\left[x^{*}, x\right] \text { as } n \rightarrow \infty,
$$

which contradicts (5).

(ii) Let $x_{n}^{*} \stackrel{d^{*}}{\rightarrow} 0$. Since

$$
\left\|x_{n}^{*}\right\|=\sup \left\{\left[x_{n}^{*}, x_{n}\right]:\left\|x_{n}\right\|=1\right\},
$$

there exists a sequence $\left(x_{n}\right)$ with $\left\|x_{n}\right\|=1$ and

$$
\left|\left\|x_{n}^{*}\right\|-\left[x_{n}^{*}, x_{n}\right]\right|<\text {, for all } n .
$$

By similar arguments as in the proof of $(i)$, there is a subsequence, again denoted by $\left(x_{n}\right)$, such that

$$
E_{n} K_{n} x_{n} \stackrel{w}{\rightarrow} W(x) \text { in } F \text { as } n \rightarrow \infty
$$


Since $x_{n}^{*} \stackrel{d^{*}}{\rightarrow} 0$, this implies that

$$
\left[x_{n}^{*}, x_{n}\right] \rightarrow 0 \text { as } n \rightarrow \infty,
$$

and hence

$$
\left\|x_{n}^{*}\right\| \rightarrow 0 \text { as } n \rightarrow \infty \text {. }
$$

Proof of Theorem 2.1: To prove $(\mathrm{C} 3) \Rightarrow(\mathrm{C} 2)$, we first show that, for fixed $b_{n} \in X_{n}^{*}$, the equation $A_{n} x_{n}=b_{n}$ has exactly one solution $x_{n} \in X_{n}$ for all $n \geq n_{0}$.

Since the operator $A_{n}: X_{n} \rightarrow X_{n}^{*}$ is injective, by the stability condition (A2), the set $A_{n}\left(X_{n}\right)$ is open by the Brouwer theorem on the invariance of domain ([6], Theorem 16C). Next, to show that the set $A_{n}\left(X_{n}\right)$ is closed, let $A_{n} x_{k} \rightarrow z$ as $k \rightarrow \infty$. Then $\left(A_{n} x_{k}\right)$ is a Cauchy sequence in $X_{n}$. It is easy to see that $\left(x_{k}\right)$ is also a Cauchy sequence, by the stability condition (A2), in $X_{n}$. Hence, $x_{k} \rightarrow x$ as $k \rightarrow \infty$. Since $A_{n}$ is continuous, we get $A_{n} x=z$, that means, $z \in A_{n}\left(X_{n}\right)$. To this end, since the nonempty set $A_{n}\left(X_{n}\right)$ is both open and closed, this implies that $A_{n}\left(X_{n}\right)=X_{n}^{*}$.

Second, we proceed to show, for fixed $b \in X^{*}$, that the equation $A x=b$ has at most one solution $x \in X$. Let us assume $A x=A y$. Then, by the stability condition (A2), we obtain

$$
\mu\left(\left\|R_{n} x-R_{n} y\right\|\right) \leq\left\|A_{n} R_{n} x-A_{n} R_{n} y\right\| \text { for all } n .
$$

By the weak consistency condition (A1), we get

$$
A_{n} R_{n} x-A_{n} R_{n} y \stackrel{d^{*}}{\rightarrow} 0,
$$

and by Lemma 2.4(ii), we have

$$
\left\|A_{n} R_{n} x-A_{n} R_{n} y\right\| \rightarrow 0 \text { as } n \rightarrow \infty .
$$

Thus,

$$
\mu\left(\left\|R_{n} x-R_{n} y\right\|\right) \leq\left\|A_{n} R_{n} x-A_{n} R_{n} y\right\| \rightarrow 0 \text { as } n \rightarrow \infty .
$$

This implies that

$$
\left\|R_{n} x-R_{n} y\right\| \rightarrow 0 \text { as } n \rightarrow \infty \text {. }
$$

It follows that

$$
\left\|E_{n} K_{n} R_{n} x-E_{n} K_{n} R_{n} y\right\| \leq\left(\sup \left\|E_{n}\right\|\right)\left(\sup \left\|K_{n}\right\|\right)\left\|R_{n} x-R_{n} y\right\| \rightarrow 0 \text { as } n \rightarrow \infty,
$$

and by the compatibility condition (I1),

$$
W(x-y)=0
$$

that is, $x=y$.

Third, we show that, for $b \in X^{*}$, the equation $A x=b$ has exactly one solution $x \in X$. Let us choose a sequence $\left(b_{n}\right)$ such that $b_{n} \stackrel{d^{*}}{\rightarrow} b$ as in (A3), $A_{n} x_{n}=b_{n}$ as in the first part of the proof. Then it follows from (A1) and Lemma $2.4(i)$ that 


$$
A_{n} R_{n}(0) \stackrel{d^{*}}{\rightarrow} A(0) \text { and } \sup \left\|A_{n} R_{n}(0)\right\|<\infty
$$

Since $R_{n}(0)=0$, we have

$$
\begin{gathered}
\left\|b_{n}\right\|=\left\|A_{n} x_{n}\right\| \geq\left\|A_{n} x_{n}-A_{n}(0)\right\|-\left\|A_{n} R_{n}(0)\right\| \\
\geq \mu\left(\left\|x_{n}\right\|\right)-\left\|A_{n} R_{n}(0)\right\| .
\end{gathered}
$$

This implies that

$$
\sup \mu\left(\left\|x_{n}\right\|\right)<\infty \Rightarrow \sup \left\|x_{n}\right\|<\infty .
$$

Since $A$ is $A$-proper, we obtain

$$
x_{n^{\prime}} \stackrel{d}{\rightarrow} x \text { and } A x=b .
$$

Fourth, we show that $b_{n} \stackrel{d^{*}}{\rightarrow} b$ and $A_{n} x_{n}=b_{n}$ imply that

$$
x_{n} \stackrel{d}{\rightarrow} x \text { and } E_{n} K_{n} x_{n} \rightarrow W(x) \text { in } F \text {. }
$$

It follows from the preceding part that each subsequence $\left(x_{n^{\prime}}\right)$ of $\left(x_{n}\right)$ has another subsequence $\left(x_{n^{\prime \prime}}\right)$ such that

$$
x_{n^{\prime \prime}} \stackrel{d}{\rightarrow} x \text { and } A x=b
$$

The limit element $x$ remains the same for all subsequences since $A x=b$ has exactly one solution $x$. Thus, the convergence of the whole sequence follows, that is,

$$
x_{n} \stackrel{d}{\rightarrow} x
$$

Finally, we show that

$$
x_{n} \stackrel{d}{\rightarrow} x \Rightarrow E_{n} K_{n} x_{n} \rightarrow W(x) \text { in } F .
$$

Since $x_{n} \stackrel{d}{\rightarrow} x$ and $\pi_{0}$ is an admissible external approximation scheme, we get, as $n \rightarrow \infty$,

$$
\begin{aligned}
& \left\|E_{n} K_{n} x_{n}-W(x)\right\|=\left\|E_{n} K_{n} x_{n}-E_{n} K_{n} R_{n} x+E_{n} K_{n} R_{n} x-W(x)\right\| \\
& \leq\left\|E_{n} K_{n} x_{n}-E_{n} K_{n} R_{n} x\right\|+\left\|E_{n} K_{n} R_{n} x-W(x)\right\| \\
& \leq\left(\sup \left\|E_{n}\right\|\right)\left(\sup \left\|K_{n}\right\|\right)\left\|x_{n}-R_{n} x\right\|+\left\|E_{n} K_{n} R_{n} x-W(x)\right\| \rightarrow 0 .
\end{aligned}
$$

The proof of $(\mathrm{C} 2) \Rightarrow(\mathrm{C} 1)$ is trivial.

Finally, we prove: $(\mathrm{C} 1) \Rightarrow(\mathrm{C} 3)$. We denote the subsequence of a sequence $\left(x_{n}\right)$, again by $\left(x_{n}\right)$. Let

$$
A_{n} x_{n} \stackrel{d^{*}}{\rightarrow b} \text { with } \sup _{n}\left\|x_{n}\right\|<\infty
$$

We further choose a point $x \in X$ with $A x=b$ as in (C1). It suffices to show that 


$$
x_{n} \stackrel{d}{\rightarrow} x
$$

that is, the condition (C3) holds. By (A1), we have

$$
A_{n} R_{n} x \stackrel{d^{*}}{\rightarrow} A x
$$

It follows that

$$
A_{n} x_{n}-A_{n} R_{n} x \stackrel{d^{*}}{\rightarrow} 0
$$

and by Lemma 2.4(ii),

$$
\left\|A_{n} x_{n}-A_{n} R_{n} x\right\| \rightarrow 0 \text { as } n \rightarrow \infty .
$$

Then, by the stability condition (A2), we get, as $n \rightarrow \infty$,

$$
\mu\left(\left\|x_{n}-R_{n} x\right\|\right) \leq\left\|A_{n} x_{n}-A_{n} R_{n} x\right\| \rightarrow 0 .
$$

This implies that

$$
\left\|x_{n}-R_{n} x\right\| \rightarrow 0 \text { as } n \rightarrow \infty
$$

that is,

$$
x_{n} \stackrel{d}{\rightarrow} x
$$

This completes the proof.

Theorem 2.5: Let $\pi_{0}=\left\{X, F, X_{n}, X^{*}, X_{n}^{*}, A, W, A_{n}, R_{n}, K_{n}, E_{n}\right\}$ be an admissible external approximation scheme represented by the diagram (1). If $X_{0}$ is dense in $X$, then

$$
E_{n} K_{n} R_{n} x \rightarrow W(x) \text { for all } x \in X_{0}
$$

implies that

$$
E_{n} K_{n} R_{n} x \rightarrow W(x) \text { for all } x \in X \text {. }
$$

Proof: Let $E_{n} K_{n} R_{n} x \rightarrow W(x)$ as $n \rightarrow \infty$ for all $x \in X_{0}$, where $X_{0}$ is dense in $X$. We need to show that, for all $y \in X$,

$$
E_{n} K_{n} R_{n} y \rightarrow W(y) \text { as } n \rightarrow \infty \text {. }
$$

Let $y \in X$ and $\epsilon>0$ be fixed. Then

$$
\begin{gathered}
\left\|E_{n} K_{n} R_{n} y-W(y)\right\| \leq\left\|E_{n} K_{n} R_{n} y-E_{n} K_{n} R_{n} x\right\|+\left\|E_{n} K_{n} R_{n} x-W(x)\right\|+\|W(x)-W(y)\| \\
\left.\leq \sup _{n}\left\|E_{n}\right\|\left\|K_{n}\right\|\left\|R_{n}\right\|\right)\|y-x\|+\left\|E_{n} K_{n} R_{n} x-W(x)\right\|+\|W\|\|y-x\| \\
\left.=\sup _{n}\left\|E_{n}\right\|\left\|K_{n}\right\|\left\|R_{n}\right\|+\|W\|\right)\|y-x\|+\left\|E_{n} K_{n} R_{n} x-W(x)\right\| \\
<\epsilon \text { for all } n \geq n_{0}(\epsilon),
\end{gathered}
$$


where $x \in X_{0}$ is so chosen that $\|y-x\|$ is sufficiently small. This competes the proof.

\section{Application}

Let us consider the following external approximation scheme $\pi_{2}=\left\{X, F, X_{n}, X^{*}, A, W\right.$, $\left.A_{n}, R_{n}, K_{n}, E_{n}\right\}:$

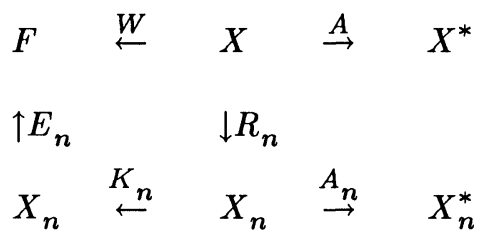

where $X=\stackrel{\circ}{W}_{p}^{1}(G), X_{n}=\stackrel{\circ}{W}_{p}^{1}\left(g_{h_{n}}\right)$, the Sobolev spaces, and $F=\prod_{i=1}^{N} L_{p}(G), 2 \leq p<\infty$. Here $G$ is a bounded region in $\mathbb{R}^{N} N \geq 1$, with sufficiently smooth boundary, that is, $\delta G \in C^{0,1}$. A sufficiently small positive number $h_{0}$ is chosen so that the set $g_{h}$ of interior lattice points is not empty for all $h, 0 \leq h \leq h_{0}$. Furthermore, $\bar{f}_{h}(P)$ represents the integral mean value of $f$ over the cube $c_{h}(P)$ belonging to $P$, that is,

$$
\bar{f}_{h}(P)=h^{-N} \int_{c_{h}(P)} f(t) d t .
$$

The operators $W: X \rightarrow F, R_{n}: X \rightarrow X_{n}, K_{n}: X_{n} \rightarrow X_{n}$ and $E_{n}: X_{n} \rightarrow F$ are defined as follows:

$$
\begin{gathered}
W(x)=\left(x, D_{1} x, \ldots, D_{N} x\right), \\
\left(R_{n} x\right)(P)=\left\{\begin{array}{cc}
k^{-N} \int_{c_{h}(P)} x(t) d t & \text { for } P \in g_{k, 1} \\
0 & \text { for } P \notin g_{k, 1},
\end{array}\right.
\end{gathered}
$$

and

$$
E_{n} K_{n} x_{n}=\left(x_{n}, \nabla_{1} x_{n}, \ldots, \nabla_{N} x_{n}\right)
$$

Now, we can apply Theorem 2.1 , for example, to the boundary value problem

$$
\left\{\begin{array}{cc}
-\sum_{i=1}^{N} D_{i}\left(\left|D_{i} x\right|^{p-2} D_{i} x\right)+s x=f & \text { on } G, \\
x=0 & \text { on } \delta G,
\end{array}\right.
$$

with corresponding difference equations

$$
\left\{\begin{array}{cc}
-\sum_{i=1}^{N} \nabla_{i}^{-}\left(\left|\nabla_{i} x_{h}(P)\right|^{p-2} \nabla_{i} x_{h}(P)\right)+s x_{h}(P)=\bar{f}_{h}(P) & \text { for all } P \in g_{h} \\
x_{h}(P)=0 & \text { for all } P \in \delta g_{h} .
\end{array}\right.
$$




\section{References}

[1] Petryshyn, W., Nonlinear equations involving noncompact operators, Nonlinear Functional Analysis 18 (Presented at the Proc. Sympos. Pure Math., Chicago 1968), Amer. Math. Soc., Providence, RI (1970), 206-233.

[2] Petryshyn, W., Projection methods in nonlinear numerical functional analysis, J. Math. Mech. 17:4 (1967), 353-372.

[3] Schumann, R. and Zeidler, E., The finite difference method for quasilinear elliptic equations of order 2m, Numer. Funct. Anal. Optimiz. 1 (1979), 161-194.

[4] Verma, R. and Debnath, L., Phi-pseudo-monotonicity and approximation-solvability of nonlinear equations, Appl. Math. Lett. 4:6 (1991), 73-75.

[5] Verma, R., Phi-stable operators and inner approximation-solvability, Proc. Amer. Math. Soc. 117:2 (1993), 491-499.

[6] Zeidler, E., Nonlinear Functional Analysis and its Applications I, Springer-Verlag, New York 1986. 


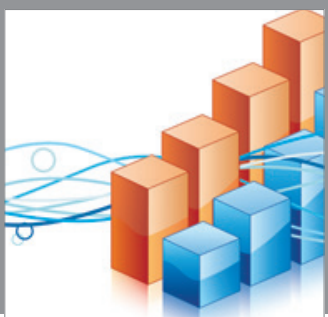

Advances in

Operations Research

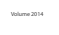

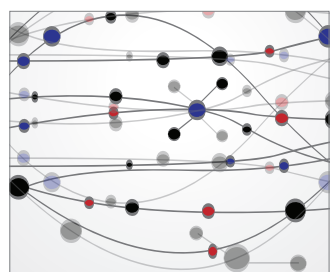

\section{The Scientific} World Journal
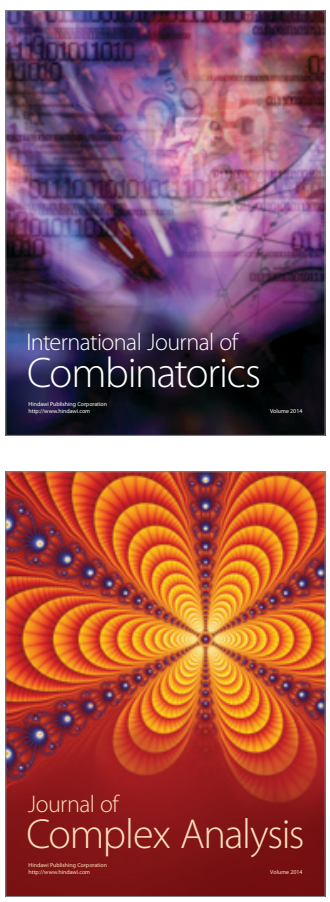

International Journal of

Mathematics and

Mathematical

Sciences
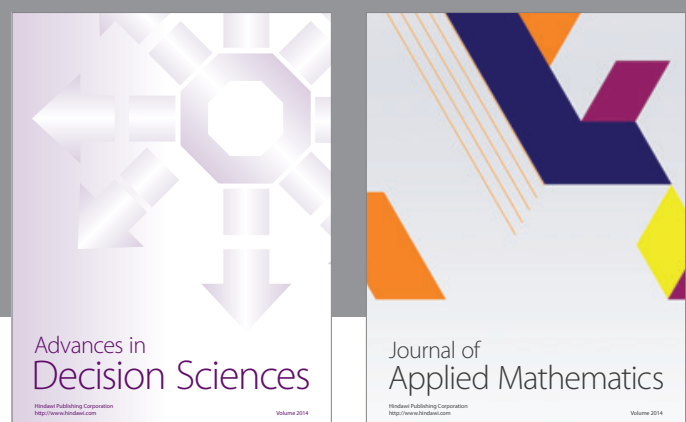

Journal of

Applied Mathematics
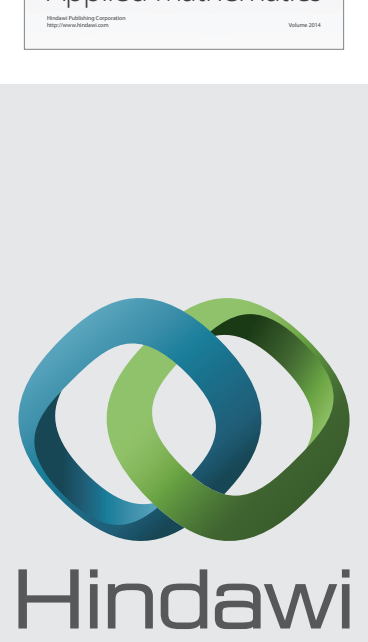

Submit your manuscripts at http://www.hindawi.com
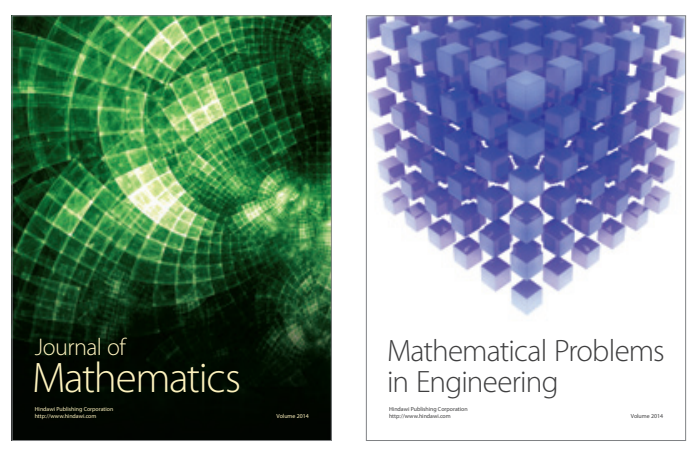

Mathematical Problems in Engineering
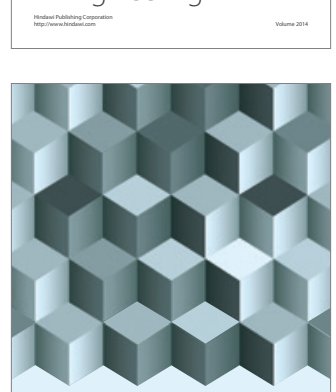

Journal of

Function Spaces
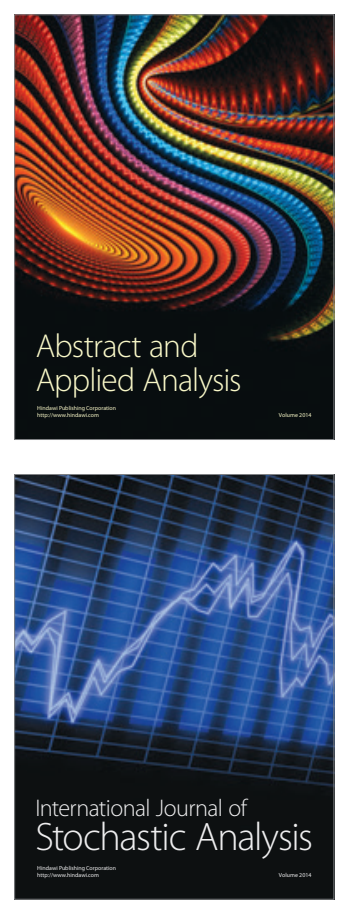

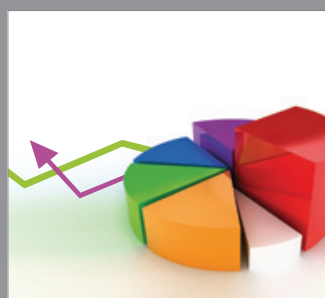

ournal of

Probability and Statistics

Promensencen
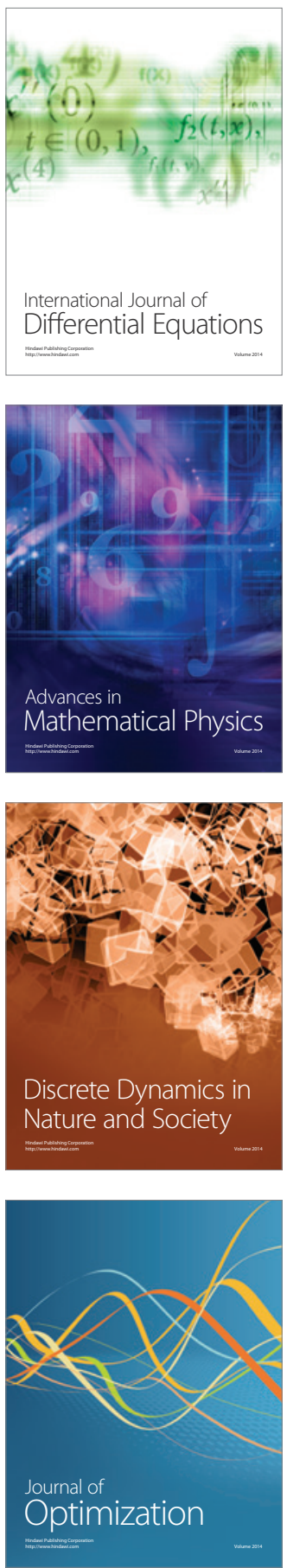